\title{
Comparative Performance Analysis of Efficient MIMO Detection Approaches
}

\author{
Muhammad Faisal, Fazal Wahab Karam, Ali Zahir \\ Department of Electrical Engineering \\ COMSATS University Islamabad, Abbottabad Campus \\ Abbottabad, Pakistan
}

\author{
Sajid Bashir \\ Department of Electrical Engineering \\ National University of Technology Islamabad \\ Islamabad, Pakistan
}

\begin{abstract}
The promising massive level MIMO (multipleinput-multiple-output) systems based on extremely huge antenna collections have turned into a sizzling theme of wireless communication systems. This paper assesses the performance of the quasi optimal MIMO detection approach based on semidefinite programming (SDP). This study also investigates the gain obtained when using SDP detector by comparing Bit Error Rate (BER) performance with linear detectors. The near optimal Zero Forcing Maximum Likelihood (ZFML) is also implemented and the comparison is evaluated. The ZFML detector reduces exhaustive ML searching using multi-step reduced constellation (MSRC) detection technique. The detector efficiently combines linear processing with local ML search. The complexity is bounded by maintaining small search areas, while performance is maximized by relaxing this constraint and increasing the cardinality of the search space. The near optimality of SDP is analyzed through BER performance with different antenna configurations using 16-QAM signal constellation operating in a flat fading channel. Simulation results indicate that the SDP detector acquired better BER performance, in addition to a significant decrease in computational complexity using different system/antenna configurations.
\end{abstract}

Keywords-Multiple input multiple output antennas; MIMO detection approaches; performance analysis; semi-definite programming; zero forcing maximum likelihood

\section{INTRODUCTION}

Wireless communication technology has seen rapid developments and unprecedented growth in the fields of computing and communication technologies during the last few decades. Wireless communication provides voice, video and data services. However, emerging services in the wireless communication are demanding more efficient network channels, high-bit rate, quality of service and higher network capacity [1]. The multimedia information traffic conveyed through the global mobile networks have been gigantic [2], [3], furthermore this tendency is put to continue, the same as suggested by VNI (Cisco- visual-networking-index) estimation [4].

Moreover, as forecast in Fig. 1 it will raise almost seven times which convert toward a CAGR (Compound-AnnualGrowth Rate) of 53\% in the time across 2017-2020, achieving 30.6 EB per month by 2020 [4]. As shown in Fig. 2. This blazing development is primarily stimulated by the dominance of mobile phones \& gadget, tablets \& laptops, and the materialization of machine-to-machine (M2M) communications [5]-[7].
Consequently, these systems have moved from SingleInput Single-Output (SISO) antenna technology to more efficient MIMO antenna technology for higher data rate and spectrally efficient wireless channels, without escalating the bandwidth or transmission power of system. However, an efficient MIMO system requires significant effort for designing efficient detectors with low computational cost. It improves data rates through spatial multiplexing and Bit Error Rate (BER) performance through diversity, which uses different detecting algorithms to decode received vectors [8].

A problem encountered in the design of optimal detector is to detect original transmitted signal (information) from noisy and faded channel in digital communication systems. In any practical scenario of information exchange between the transceivers, the designing of detector poses a big challenge to meet specifications such as minimal probability errors, computational efficiency and less complexity. Unfortunately, such type of detectors is computationally complex and often left out in favor of sub-optimal detectors. However, in many cases, the performance is considerably different during analysis of suboptimal and optimal detectors. On the other hand, the computationally efficient, cost effective optimal detection makes the optimal detectors attractive in comparison to its counterpart.

The Maximum Likelihood (ML) detector gives minimum error probability [9] but it is impractical to use higher-order modulation (16-QAM) in MIMO systems due to its exhaustive search requirements. Different linear, sub-optimal and near optimal, detectors are generally discussed to reduce the ML complexity. These detectors are commonly known as Sphere Detectors that provide optimal performance with reduced com-

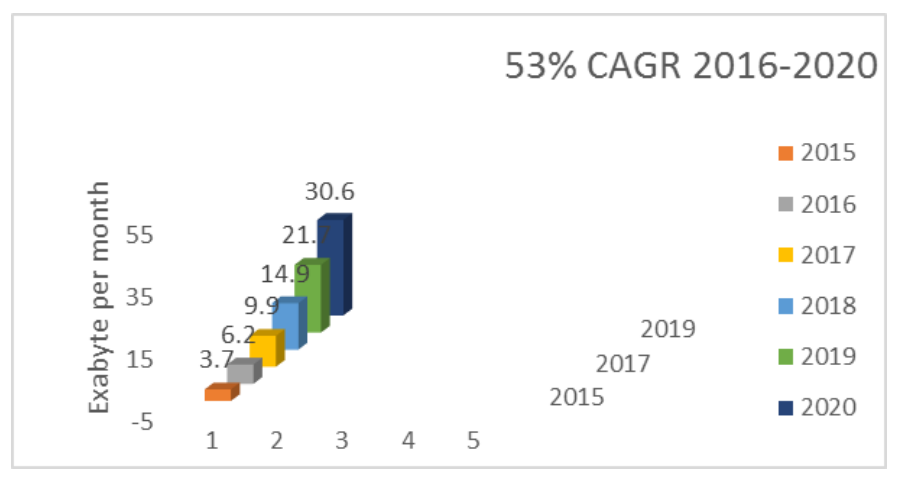

Fig. 1. Cisco VNI 2016-2020 traffic forecast for worldwide mobile data 


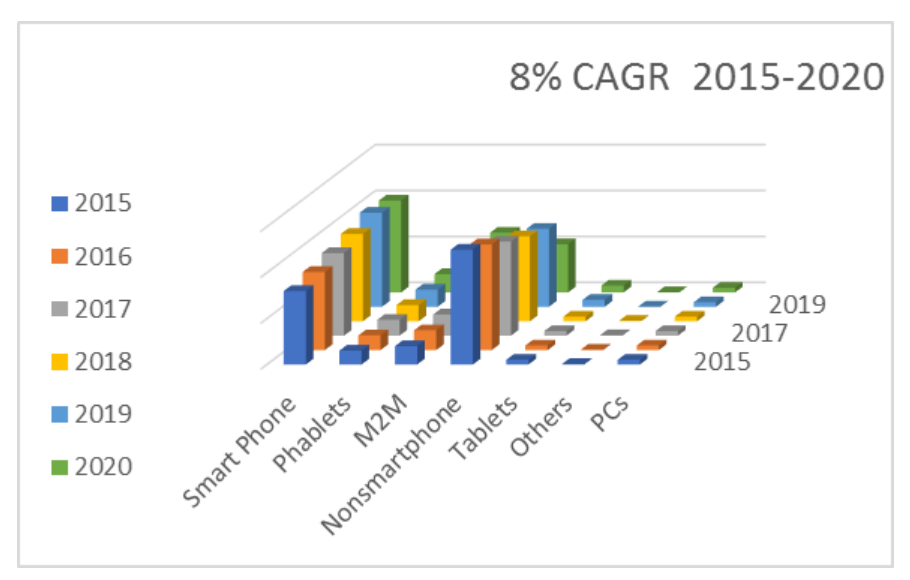

Fig. 2. Cisco VNI: Global mobile devices and connections growth forecast, 2015-2020.

putational complexity [10]. Zero Forcing (ZF) detector [11] is suboptimal linear detector. It has a polynomial complexity of cubic order $O\left(n^{3}\right)$ for $M_{t} \times N_{t}$ MIMO systems. It forms the computational complexity of computing the pseudo-inverse of the matrix channel. The linear, suboptimal and near optimal detectors are computationally less complex. However, the compromise is the degraded BER performance in comparison to the ML detector. Presently, computationally efficient and lesser complex high-performance MIMO detector such as ZFML [12] is near optimal heuristic detector. It reduces exhaustive ML searching using MSRC detection technique. However, the performance of ZFML detector is better in large search space.

The designing of detection poses a big challenge to meet specifications such as minimal probability errors, computationally efficient and reduce complexity. The use of semi-definite relaxation (SDR) offers efficient, high-performance detection approach [13]. SDR is efficient in solving the computationally complex ML detection problem and numerous detection problems that are discussed in [14].

The work [15] formulated ML problem in a higher dimension to relax rank-1 constraint (non-convex problem to convex problem) and derived as SDR. It provides better solution in computational complex problems [16].

\section{Multiple InPut Multiple Output System Model}

MIMO system gives significant improvement in spectral efficiency of wireless channel without escalating the bandwidth or transmission power of system. However, an efficient MIMO system requires significant effort for designing efficient detectors with low computational cost.

The block diagram in Fig. 3 depicts complex MIMO system [17]. The data bits are encoded and interleaved for transmission. The data symbols (QAM symbols) are mapped through interleave code-words; space-time encoder seizes data symbols and generates spatial data streams. The Space-Time Block-Encoder map the spatial streams and then transmit information signal to the receiver, subsequently received vector is decoded, de-mapped and de-interleaved.

The narrowband MIMO channel consists of point-topoint wireless system of $M_{t}$ "transmit $\left(T_{x}\right)$ " and $N_{r}$ "receive $\left(R_{x}\right)$ " $\left(N_{r}>M_{t}\right)$ as shown in Fig. 4 where $M_{t}$ input symbols $S_{t}=\left[S_{1} \ldots S_{M}\right],\left(n_{1} \times 1\right)$, transmitted during the $j^{\text {th }}$ time slot. $N_{r}$ is having receive vectors $y_{r}=\left[y_{1} \ldots y_{r}\right]$, $\left(n_{1} \times 1\right)$. Noise is denoted as $n=\left[n_{1} \ldots n_{r}\right],\left(n_{1} \times 1\right)$ containing AWGN elements with $\sigma^{2} n$ variance. $H=\left[h_{1} \ldots h_{N}\right]$ denotes complex $M_{t} \times N_{r}$, channel matrix, the $i^{\text {th }}$ column and $i^{t h}$ row is $h_{i j}$. Complex Gaussian is Rayleigh distribution that depicts flat fading channel.

MIMO techniques as shown in Fig. 6 are used in technologies e.g. Wi-Fi and LTE, and emerging techniques e.g. LTE Advanced. Comparing the performance while applying multiple-input-multiple-output (MIMO) techniques particularly, several setups with various MIMO algorithms are considered.

\section{A. MIMO Detection Approaches and Challenges}

As indicated by Shannon "Primary dilemma in communication is to replicate at some point both precisely \& roughly a signal chosen on a different spot" [18].

Comparatively to typical single-input and single-output (SISO) systems, MIMO systems contain multiple interfering symbols/messages conveyed simultaneously, furthermore, subsequently these messages/symbols are anticipated to be decoded /detected at the receiver pertaining to corruption by haphazard interference/noise as presented in Fig. 5. The compound messages/symbols might be sensed/ detected alone otherwise mutually. Contrasting to alone sensing/detection, every symbol/message has to be sensed/detected considering the uniqueness of the other messages/symbols in mutual sensing/detection. Since a useful outcome, characteristically mutual detection is able of achieving a considerably superior efficiency than alone detection/sensing, even though mutual sensing/detection inflicts higher computational complexity.

The mutual sensing/detection of compound messages in Multiple Input Multiple Output systems is of fundamental significance for the purpose of grasping the important benefits of diverse Multiple Input Multiple Output methods. Due to the CCI (co-channel-interference) usually encounter in Multiple Input Multiple Output systems make up primary restrictive feature [19]-[21]. Desolately, the best possible MIMO detection issue is established as non-deterministic polynomial-time hard (NP-hard) [22], [23]. Consequently all well-known algorithms considered for resolving the problem for optimal solutions,

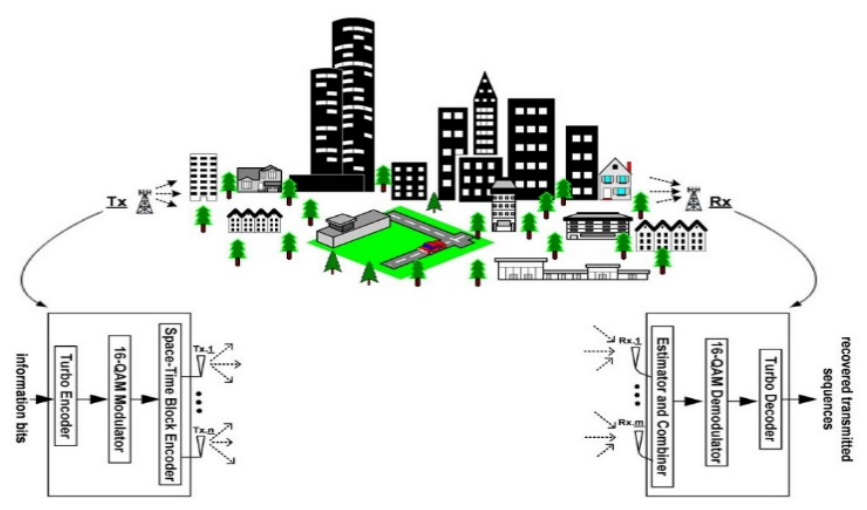

Fig. 3. Block diagram of Complex MIMO system. 


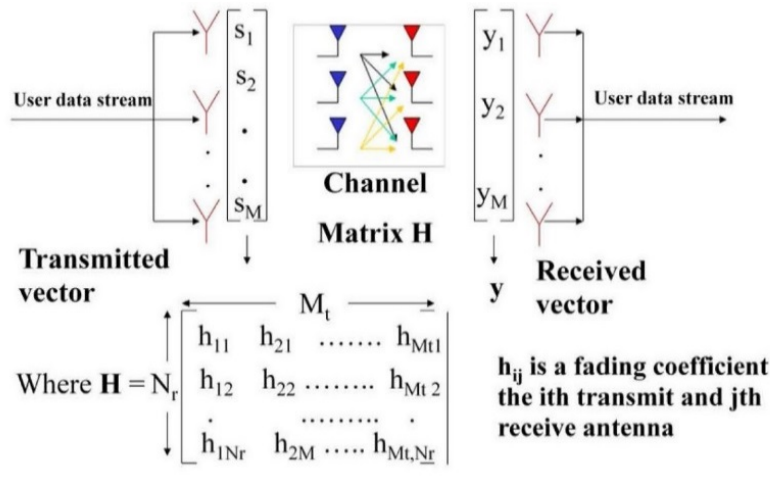

Fig. 4. MIMO channel "where $M_{t}$ represents number of "transmit ( $\left.T_{x}\right)$ ") and $N_{r}$ "receive $\left(R_{x}\right)$ " antenna correspondingly".

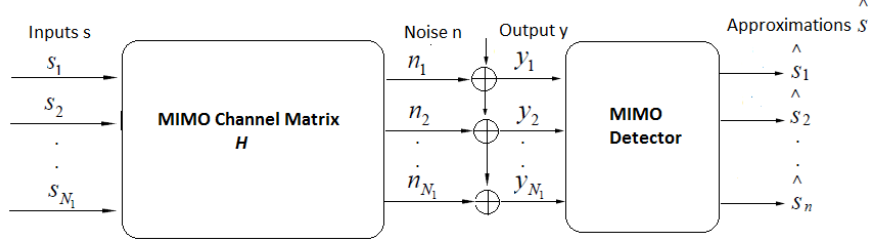

Fig. 5. MIMO detection dilemma.

incorporate exponential rise of complexity with the raise in number of decision factors. As a result, the computational complexity of the best possible ML (maximum-likelihood) condition based MIMO detection algorithms rapidly turn into redundant as the numbers of decision variables are augmented. Practically all modern ICs meet an integration density margin due to the maximum bearable internal temperature forced by the extreme power consumption, resulting restriction on IC development. As a result, one may not merely depends on Moores law. Moreover yet mild complex Multiple Input Multiple Output sensing/detection methods might be excessively power starving designed for systems based on battery. Therefore modest complexity, however superior-performance suboptimum Multiple Input Multiple Output detection procedures are required intended for realistic Multiple Input Multiple Output-applications.

Spatial multiplexing methods concurrently transmit selfdetermining information sequences, frequently known layers, using multiple antennas. With an $\mathrm{M}$ transmit antennas, in general bit rate contrast to a single-antenna system is improved by a factor of $\mathrm{M}$ with no requirement for additional bandwidth or further transmission power. Channel coding is frequently engaged, hence to warranty a definite error performance. As the individual layers are super-imposed throughout communication, need to be alienated at the receiver by an interference cancellation category of algorithm (classically in combination with multiple receive antennas). A renowned spatial multiplexing method is the BLAST (Bell-Labs Layered Space-Time Architecture). The realized bit rate comparing to a singleantenna system is known multiplexing gain e.g. an antenna gain, multiplexing gain and diversity gain.

\section{B. The multiplexing gain}

The truth to facilitate the capacity of a MIMO system with $\mathrm{M}$ transmit and $\mathrm{N}$ receive antennas raises (more or less) linearly with the minimum of $\mathrm{M}$ and $\mathrm{N}$ (exclusive of entailing further bandwidth or additional transmission power) is an fascinating outcome. For SISO, setting a predetermined bandwidth, capacity may barely be improved logarithmically with the SNR, by rising the transmit power. TIn [1], the theoretical capacity outcomes for MIMO systems were matched by the scheme of the BLAST method, achieving bit rates approximately $90 \%$ of outage capacity. The first real-time BLAST demonstrator was set with $\mathrm{M}=8$ transmit and $\mathrm{N}=12$ receive antennas achieving exceptional bit rates of $40 \mathrm{bit} / \mathrm{s}$ per Hertz in contrast to any SISO system.

\section{Spatial Diversity}

Multiple antennas may also be used to enhance the error rate of a system (error performance), as a result of transmitting and receiving unneeded signals presenting the identical information sequence accommodating in the spatial domain, rather than in the time domain without lowering the effective bit rate in contrast to single-antenna transmission. Spatial diversity methods primarily aim at an enhanced error performance in contrast to spatial multiplexing schemes based on a diversity gain and a coding gain. Two forms of spatial diversity as macroscopic and microscopic diversity can be found in a comprehensive survey of spatial diversity for wireless communication systems [20].

\section{Signal-to-noise Ration and Co-Channel Interference}

In addition to higher bit rates and smaller error rates, multiple-antenna techniques may also be employed to enhance the SNR at the receiver and to contain co-channel interference in a multiuser situation by adopting smart antennas or software antennas. Beam-forming schemes which are interpreted as linear filtering in the spatial domain are employed, the beam patterns of the transmit and receive antenna array may be steered in particular preferred directions, whereas un-preferred directions having significant interference may be nulled.

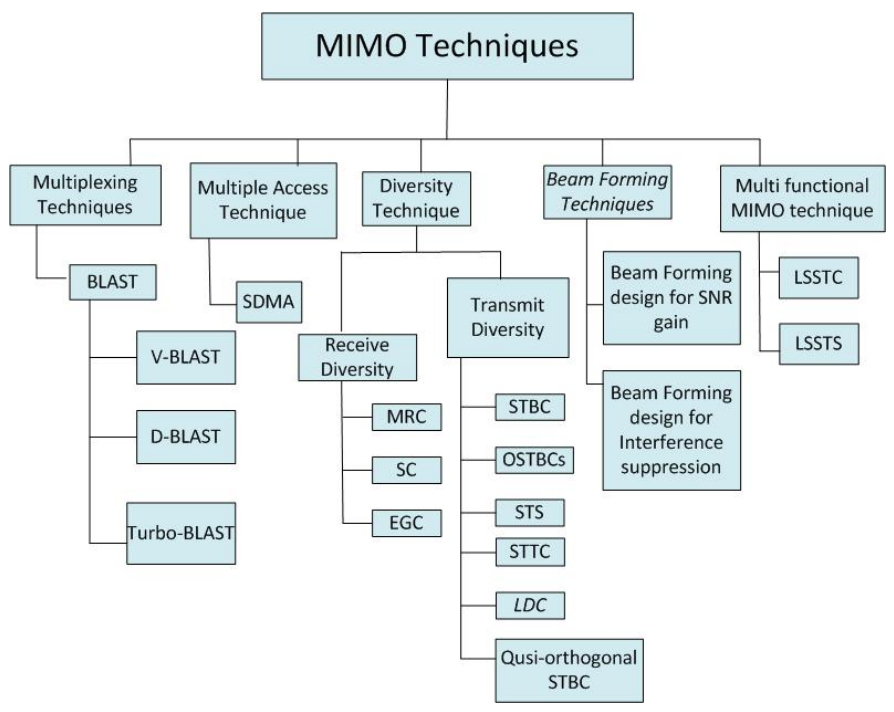

Fig. 6. MIMO Techniques. 


\section{E. Smart antennas and beamforming Schemes}

Beam-forming schemes may be used to achieve enhanced data rates and better error rates causing better SNR at the receiver and suppressed co-channel interference $(\mathrm{CCI})$ in a multiuser scenario. As the spectrum is restricted, so the sharing is needed to enhance the capacity of cell by allocating the available bandwidth at the same time to multiple users using multiple access methods while maintaining the quality of service trade within the existing users. SDMA employed the spatial separation of the mobile users to enhance the use of the frequency spectrum. The transmission power of every user is restricted by Space division multiple access using spot beam antenna serving by the similar frequency or diverse frequencies. TDMA or CDMA is employed by the antenna beam covering diverse areas, frequency may be re-used, in, for diverse frequencies FDMA may be employed. Multi-functional MIMO mingle the benefits of numerous MIMO methods, e.g. multiplexing gains, diversity gains, and beam-forming gains, e.g. V-BLAST is able of realizing maximum possible multiplexing gain, while STBC may realize the full possible antenna diversity. In V-BLAST and STBC is combined to offer together antenna diversity and spectral efficiency gain. Additionally, combined array processing is enhanced through enhancing the decoding order of the different antenna layers. The optimized receive diversity gain for the mutual VBLAST- STBC system assisted by the number of separately fading diversity channels is achieved in an iterative decoding algorithm. A transmission scheme known as D-STTD (double space-time transmit diversity), comprising of two STBC layers at the transmit antennas, whereas the receiver is prepared with two antennas is presented. Beam-forming has pooled both spatial diversity and spatial multiplexing schemes to realize extra performance gain e.g. beam-forming and STBC has been pooled together.

\section{Multiple Input Multiple Output SENSING/DETECTION DiLEMMA}

Regardless of the reality that related problems have been identified for a while [22], [24], [25] the idiom "MIMO detection" became common primarily with the advent of multipleantenna systems throughout the mid-1990s [26], [27]. As a consequence, in the common sense, Multiple Input Multiple Output detection typically applied on to the symbol detection issue materialized in narrow-band SDM based multiple antenna methods, e.g. VBLAST (Vertical Bell Laboratories layered space-time system) [28]. Though, it is emphasized to facilitate a group of significant signal processing methods, Multiple Input Multiple Output detection ought to be understood based on a general mathematical model. In the broad sense, the MIMO detection issue can be characterized for an $M_{t}$ input linear scheme whose transfer function is expressed by a matrix, containing non-orthogonal columns and Its output $N_{r}$ is corrupted by additive random interference, which does not essentially comply with the Gaussian distribution. The compound inputs may be represented like a vector $S$ that is arbitrarily retrieved from the set $S^{M_{t}}$ complied by $M_{t}$ element vectors, whose elements are drawn from a finite set $S_{t}=\left[S_{1} \ldots S_{M}\right]$. The "deductive" or "presumptive" probability of picking each vector from $S^{M_{t}}$ is similar. The set is generally referred as the constellation alphabet, whose components may get any complex or real values. Furthermore, $S_{n}, n=\left[n_{1} \ldots n_{r}\right]$, correspond to the realizations of $S$, therefore these are the components of $S^{M_{t}}$. Next the relationship among the inputs and the outputs of this linear scheme may be described by

$$
Y=H s+n
$$

where $Y$ is receive signals vector, $H$ is channel matrix of the system, and $n$ additive noise is denoted by $\sigma^{2} n$ containing AWGN elements with variance. Based on the particular applications it may be moreover the field of $\mathbb{R}$ (real numbers) or the field of $\mathbb{C}$ (complex numbers). In brief, every scheme having compound inputs \& outputs, and pertaining to additive random interference may be considered as a Multiple Input Multiple Output system, however the MIMO detection issue concerned in MIMO systems, is simply just tackled whose channel matrix is non-orthogonal in columns. This is significant that the $s$ (constellation alphabet), the $M_{t}$ (number of inputs) and the number of outputs $N_{r}$ are usually considered as constant quantities for a particular system. Therefore, these are understood to be identified by default, though it will not be explicitly underlined, except needed. While an additional note, as the input message/symbol vectors of compound successive time-slots are linked together using space-time-coding [29], [30], the Multiple Input Multiple Output system is specified as

$$
Y=H s+n
$$

wherever $Y$ denoting a matrix indicating the message/signal received in multiple time-slots, $H$ denoting a matrix denoting the space-time codeword, and presents the resultant noise/interference matrix. Equation (1) may be realized from (2) by putting the number of time-slots regarded to one. In this context, (2) is more general than one (1), though, equation (2) is primarily employed for differentiating space-timecoding aided Multiple Input Multiple Output schemes. This is due to the best possible ML decoding/detecting could be purely implemented based on the separate symbol-by-symbol decoding approach or pair-wise decoding approach [30], [31]. Thus, majority cases related with Multiple Input Multiple Output detection, depends on the system model presented in (1). The fundamental job of Multiple Input Multiple Output detection is to approximate the key input vector based on the information of the expected/received signal vector $Y$ and the channel matrix $H$. If the instantaneous value of $H$ is eminent from precise channel estimation, the denote/detection of $s$ is said to be based on coherent detection. Even though, if the precise estimation of the instantaneous channel state is evaded, the detection of $s$ fit in to non-coherent detection scheme.

\section{Multiple Input Multiple Output Detection APPROACHES}

\section{A. Maximum Likelihood detection}

The ML detection in higher-order modulation (M-QAM) is an NP-Hard problem due to exhaustive search in MIMO systems. Therefore, it is impractical even for moderate systems. For this reason, less computationally complex and efficient detectors are needed to develop.

The transmit symbols are from a random finite alphabet or constellation $S \subset \mathbb{C}, S=S_{1} \ldots S_{M}$ of size $M_{t}$. The detector's function is to select one of the $M^{M_{t}}$ or $2^{k M}$ possible 
transmitted symbol vectors from whole set of transmitted symbols. Suppose symbol vectors $S \in S^{M_{t}}$ are equi-probable.

$$
S^{*}=\underset{s \in s^{n_{t}}}{\arg \max P(y \text { is observed } \mid \text { s.was sent })}
$$

ML detector always returns an optimal solution according to (3). Optimal detection is performed over the search space of all possible input $\mathrm{s}$ vectors. Since the search space has random integer components, this problem called least-squares optimization problem and it is non-deterministic polynomial (NP) which is time hard and Combinatorial Optimization Problems (COP). This type of problems involves an optimal solution with respect to an objective function for detection. COP use exhaustive search to enumerate optimal solutions and selecting the one which minimizes the objective function in shown in (4).

$$
S^{*}=\underset{s \in s^{n_{t}}}{\arg \min P}\|Y-H s\|^{2}
$$

The ML detector of (4) represents a discrete optimization problem over $|S|^{M_{t}}$ candidate vectors $S \in|S|^{M_{t}}$. Unfortunately, such problems are in general hard to solve and it has been shown that the problem of (4) for general $y$ and $H$ is NP-hard [23].

ML detector for $M_{t} \times N_{r}$ MIMO system with higherorder modulation (M-QAM constellation alphabet), has high computational complexity that increases exponentially with constellation size $M$ and number of transmitters $M_{t}$. A ML detector has to search $|M|^{M_{t}}$ symbols vectors. The ML computational complexity in 16-QAM and 2 transmit antennas is $|M| 2=162=256$, for 3 transmit antennas, it is $|M| 3=163=4096$ and for 4 transmit antennas, it is $|M| 4=164=65536$.

\section{B. Zero Forcing detection}

$\mathrm{ZF}$ is a suboptimal linear detector which uses MoorePenrose pseudo-inverse i.e $H^{\dagger}=(H H H)-1$. Here, $H H$ is the channel frequency response of the received signals, perfectly suppressing the Inter-Symbol Interference (ISI). For example, frequency response $F(f)$ of the detector $s(s)$ is constructed as $s(s)=1 / F(f)$. Thus, the combination of channel and equalizer gives a linear phase $F(f) s(f)=1$, meaning a flat frequency and channel response i.e., $H(s)$. Afterwards, the input signal is multiplied by the reciprocal of this. This removes the effect of ISI from the received signal.

$\mathrm{ZF}$ is successive technique to cancel interference or ISI. The interference caused by transmitted channel is then subtracted from the received signals(s). For simplicity let us consider MIMO channel modeled as in (1). To get input symbols $(s)$, we need matrix that satisfies $H^{\dagger} H=1$. The ZF detector to meet that type of constraint is given by,

$$
H^{\dagger}=\left(H^{H} H\right)^{-1} H^{H}
$$

Where $H^{\dagger}$ is Equalization Matrix and $H$ is Channel Matrix. Equation (5) is known as the Pseudo-inverse of $M_{t} \times N_{r}$ matrix. Here,

$$
\begin{aligned}
H^{H} H & =\left[\begin{array}{ll}
h_{1,1}^{*} & h_{1,2}^{*} \\
h_{2,1}^{*} & h_{2,2}^{*}
\end{array}\right]\left[\begin{array}{cc}
h_{1,1}^{*} & h_{1,2}^{*} \\
h_{2,1}^{*} & h_{2,2}^{*}
\end{array}\right] \\
& =\left[\begin{array}{cc}
\left|h_{1,1}\right|^{2}+\left|h_{2,1}\right|^{2} & h_{1,1}^{*} h_{1,2}+h_{2,1}^{*} h_{2,2} \\
h_{1,2}^{*} h_{1,1}+h_{2,2}^{*} h_{2,1} & \left|h_{1,2}\right|^{2}+\left|h_{2,2}\right|^{2}
\end{array}\right]
\end{aligned}
$$

To observe the matrix, $H^{H} H$ are not zero in off diagonal elements because the off-diagonal elements are non-zero in values. ZF detector try to null out the interfering terms when performing the detection, i.e. when solving for $s_{1}$, the interference from $s_{2}$ is tried to make it null and vice versa. ZF performs $N_{r}-M_{t}+1$ diversity order in a $M_{t} \times N_{r}$ MIMO system with $N_{r}$ possible diversity order. The ZF degrades BER performance due to noise amplification, lost whiteness property of AWGN, correlated across the data streams and is unable to detect parallel received signal. ZF detector [11] is suboptimal linear detector. It has a polynomial complexity of cubic order $O\left(n^{3}\right)$ for $n \times n$ MIMO systems. It forms the computational complexity by computing the pseudo-inverse of the matrix channel $H$.

\section{Near Optimal Heuristic Approach}

The quadratic form of (3) given as

$$
f(x)=\|y-H s\|^{2}
$$

The function $f(x)$ in (5) is convex. This near optimal Heuristic detector or algorithm reduces exhaustive ML searching and is suitable for higher order constellations. This detection algorithm also termed as multi-step reduced constellation (MSRC) detection performs local search of the target symbols within certain constraint specified reduced search space. In fact, a ZF initial solution estimate is used to define the radius of search. Constellation points around the ZF solution are searched in steps using (3) to find out the minimum Euclidian distance. This particular method which starts with the ZF processing is termed as ZFML [12].
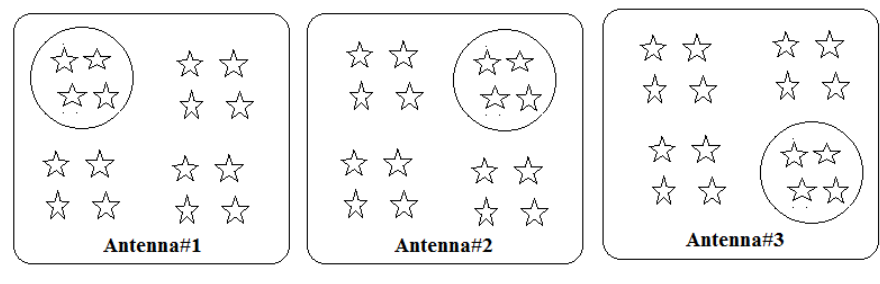

Fig. 7. ZFML reduced constellation search.

First $y$ is computed and then a ML search around the neighborhood of $y$ is performed as depict in Fig. 7. Each of the $M_{t}$ symbol generates a neighbor list, then a joint $\mathrm{ML}$ search our reduced constellations is performed. This process continues in an iterative fashion. Since the search output is generated until optimal solution is achieved. In contrast to ML search over the entire search space, the ZFML uses reduced constellation to decrease the computational complexity factor $\left(M / M_{n}\right)^{M_{t}}$ of ZFML, where $M$ constellation size is, $M_{n}$ is reduction rate and is number of transmitting antennas. If reduction rate is $|M|^{M_{t}}=16$, then after completing two rounds, the reduction rate is $16^{M_{t}} / 2$. 


\section{Sub-Optimal Approach}

Comparing to other MIMO detectors, the SDR technique is based on a respite of the best possible Multiple Input Multiple Output detection issue to the mathematical model of semidefinite programming (SDP), which is a sub-field of convex optimization [33].

Convex optimization represents a subfield of the general mathematical optimization issue. This provides study of fundamental framework as shown in Fig. 8 for minimization of a convex objective function over convex sets. It solves mathematical optimization problems by means of convex optimization which is considered as straightforward problem, due to powerful numerical algorithms, e.g. the interior-point method [35], which efficiently compute the optimal solution of convex issues. Thus, convex optimization problem is resourcefully resolved, in contrast to non-convex optimization problem which is usually tricky to work out. Convex optimization has a variety of additional vital characteristics e.g. each local optimal resolution represents the global most favorable result; consequently, there is no hazard of being misleaded by the local best possible. Furthermore, a thorough optimal situation and the duality theory is present to substantiate the best nature of a resolution in convex optimization problem [34], [36].

The SDR based MIMO detectors in recent times have received considerable research interest [37], [38]. The main striking feature of the SDR detectors is to support a polynomialtime 16 worst case computational complexity, whilst attaining a soaring performance in certain situations. SDR was first suggested for a BPSK modulated CDMA scheme [37], [39], [40], moreover next it was extended to Quadrature phase shift keying (QPSK) [41].

SDR [15] is suboptimal detection technique for higher order modulation (M-QAM) in MIMO system. SDR is efficient in solving the computationally complex ML detection problem and numerous detection problems are discussed in [42]. However, [15] formulated ML problem in a higher dimension and afterward relax rank-1 constraint (non-convex problem to convex problem) and derived as SDR, the rank relaxation method is known semi-definite programming (SDP). The SDP is better in computational complex problem and solve the problem efficiency in polynomial time [43]. The fundamental principle of SDP based detectors is demonstrated in Fig. 9, where the boxes signifies the technical challenges.

1) Rank Relaxation: There are several engineering problems having non-convex constraint such as NP-hard problems. In such problems, the non-convex constraints may be dropped or relaxed, resulting in a relaxed problem i.e. convex. Drop-

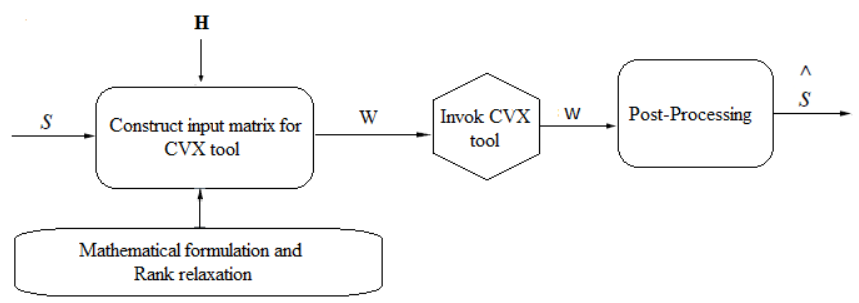

Fig. 8. Framework of solving problem using convex optimization.

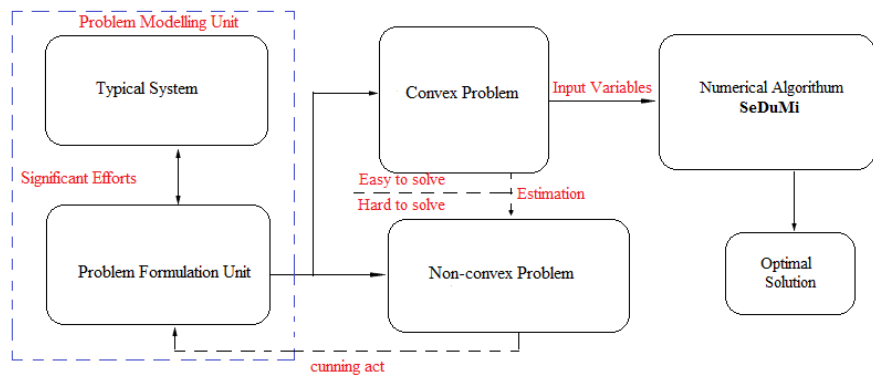

Fig. 9. The basic principle of the SDR detection technique.

ping constraints generate more feasible set to solve problem (minimize or maximize the objective function). Obviously, this feasible set gives more solutions which are not desirable in optimal solution. The reason is the relaxation of the constraints, and the set cannot be directly used as an estimated solution of the original problem, because it may not lie in the original feasible set. Thus, simple quantization, Eigen-value decomposition and randomization are used to approximate the solution [15]. Therefore, a relaxation algorithm solves the relaxed problem.

2) Semi-definite Programming Rank Relaxation: Afterwards, an approximation algorithm is used to transform a relaxed solution to an approximate one for the original problem. Considering ML problem as optimization problem in SDPRelaxation, i.e.

$$
\hat{s}_{M L}=\underset{\hat{s} \in s^{M L}}{\arg \min }\|y-H s\|^{2}
$$

The SDR attempts to estimate the solution of (8) by forming a non-convex to convex problem. The problem (8) can be expressed as:

$$
\begin{gathered}
\|y-H \hat{s}\|^{2}=\hat{s}^{T} H^{T} \hat{s}^{T}-2 y^{T} H \hat{s}+y^{T} y \\
{\left[\begin{array}{cc}
A & b \\
b^{T} & c
\end{array}\right]}
\end{gathered}
$$

Lemma: 1 Lemma: 1 let A be a symmetric matrix. The condition $s^{T} A s-2 b^{T} s+c \geq 0$ holds for all $s$ if and only if the matrix $\left[\begin{array}{cc}A & b \\ b^{T} & c\end{array}\right] \geq 0$ is semi-definite positive.

The problem (9) can be written as quadratic function using lemma\#1

$$
\left[\begin{array}{lll}
s^{T} & 0 & 1
\end{array}\right]\left[\begin{array}{ccc}
H^{T} H & 0 & -H^{T} y \\
0 & 0 & 0 \\
-y^{T} H & 0 & y^{T} y
\end{array}\right]\left[\begin{array}{l}
s \\
0 \\
1
\end{array}\right]
$$

Consider $w=\left[\begin{array}{lll}s & 0 & 1\end{array}\right]^{T}$ to form the ML problem as optimization problem

$$
\begin{aligned}
& \min w^{T} C w \\
& w \in \mathbb{R}^{m+1}
\end{aligned}
$$

where

$$
C=\left[\begin{array}{ccc}
H^{T} H & 0 & -H^{T} y \\
0 & 0 & 0 \\
-y^{T} H & 0 & y^{T} y
\end{array}\right]
$$


$s= \pm 1, \pm 3$. In symmetrical problem constraints that given by $[w]_{m+1}=1$ do not need to be enforced due to the symmetry of the problem such that if $\widetilde{x}$ is a minimizer of (12) then so is $-\widetilde{x}$. Therefore $w^{2}=1$ implies $w \in\{ \pm 1\}$. By introducing $W=w T w$ the problem can be equivalently written as optimization problem.

$$
\begin{array}{r}
\min C W \\
W, x \\
\text { s.t. } \operatorname{diag}(W)=e \\
W=w w^{T}
\end{array}
$$

Problem (8) and (14) are equal in solution.

The problem (14) also written as with the rank of $\mathrm{W}$

$$
\begin{array}{r}
\min C W \\
W \\
\text { s.t. } \operatorname{diag}(W)=e \\
\operatorname{rank}(W)=1
\end{array}
$$

since vector $e$ is all one which satisfy $e=\operatorname{diag}(W)$ factorized according to $W=w w^{T}$ for $w \in\{ \pm 1\}^{m+1}=S^{m+1}$.

Problem (8) and (15) are equal. Specifically, these problems are computationally complex and NP-hard [23] to solve. The problem (14) is non-convex problem due to rank-1 constraint. In [16] relax rank-1 constraint from $\mathrm{W}$ with a, positive semidefinite, constraint, $W \geq 0$. Now the semi-definite relaxation of (15) written as

$$
\begin{array}{r}
\min C W \\
W \\
\text { s.t. } \operatorname{diag}(W)=e \\
W \geq 0
\end{array}
$$

However, $W=w w^{T}$ implies $W \geq 0$ it follows the problem (15) shows a relaxation of (14). While $W$ have $(m+1)^{2}$ variable, as different to $m+1$ variables in $w$, the relaxation of rank-1 takes place in higher order dimensional.

The semi-definite Problem (16) has efficient methods to solve in polynomial time [46]. In particularly there are efficient techniques outlined in [15], [32], [46], [47] which solve (16) in $O\left(K^{2} N\right)$ time. If the solution of problem (16) occurred to be rank-1 then it is solved in (15). On the basis of studying the problem (16) in context of digital communication, sometimes it gives same solution of (16) is certainly of rank-1. In a scenario, when this is not the case of the solution to (15), even then this can be guaranteed to obtain from the solution of (16) having high probability. However, an efficient method for the estimation of $\hat{s}_{M L}$ is proposed for high rank solution in [42], [49] and the very method had analyzed for accuracy in the work [48].

3) Optimization problem Comprised in SDP: The problem is combinatorial problem/optimization problem with finite alphabet constraints. The problem may be solved in bruteforce fashion by searching over all the $|M|^{M}$ possible vector combination.

$$
\left\{\begin{array}{c}
\min \|y-H s\|^{2} \\
\text { s.t.s } i \in\{ \pm 1, \pm 3\}, i=1 \ldots 2 k .
\end{array}\right.
$$

The finite alphabet constraint $\pm 1, \pm 3$ can be replaced with the polynomial constrain $\left(s_{i}+1\right)\left(s_{i}-1\right)\left(s_{i}+3\right)\left(s_{i}-3\right)=0$, $i=1 \ldots 2 k$ and introduce the slack variables $t_{i}=s_{i}^{2}$ for , $i=$ $1 \ldots 2 k$ to formulate in a higher order polynomial constraint.

$$
\left\{\begin{array}{c}
\min _{s, t}\|y-H s\|^{2} \\
s . t \ldots s_{i}^{2}-t_{i}=0, i=1 \ldots 2 k \\
\ldots t_{i}^{2}-10 t_{i}+9=0, \quad i=1 \ldots 2 k
\end{array}\right.
$$

SDR detector approximates expression (18) by relaxing the feasible set of expression (17) and forming a non-convex to convex problem for optimal solution. To formulate higher dimension optimization problem, it derives the SDR and replaces $\mathrm{s}$ and $\mathrm{t}$ vectors of expression (18) with rank-1 semi-definite matrix $W=w w^{T}$, where $w^{T}=\left[s^{t} t^{T}\right]$.

The constraint easily identify $W=w w^{T}, W_{2,2}=t t^{T}$ and $W_{2,3}=t$, where $W_{i, j}$ for $i, j=1,2,3$ are the $(i, j)^{t} h$ sub-blocks $W$ of suitable sizes. However, in order to make problem in expression (18) an optimization problem we have:

$$
\begin{aligned}
\min _{w} \operatorname{Tr} & \left\{\left[\begin{array}{ccc}
H^{T} H & 0 & -H^{T} y \\
0 & 0 & 0 \\
-y^{T} H & 0 & y^{T} y
\end{array}\right]\right\} \\
& \text { s.t } \quad \operatorname{diag}\left\{W_{1,1}\right\}-W_{2,3}=0 \\
& \operatorname{diag}\left\{W_{2,2}\right\}-10 W_{2,3}+91=0 \\
& W \geq 0 \\
& W_{3,3}=1 \\
& \operatorname{rank}(W)=1
\end{aligned}
$$

The problem in expression (19) is optimization problem and non-convex problem due to rank-1 constraint. However non-convex problem in expression (19) is computationally hard to solve, so relax the constraint 1 to form convex problem:

$$
\begin{aligned}
\min _{w} \operatorname{Tr} & \left\{\left[\begin{array}{ccc}
H^{T} H & 0 & -H^{T} y \\
0 & 0 & 0 \\
-y^{T} H & 0 & y^{T} y
\end{array}\right]\right\} \\
& \text { s.t } \operatorname{diag}\left\{W_{1,1}\right\}-W_{2,3}=0 \\
& \operatorname{diag}\left\{W_{2,2}\right\}-10 W_{2,3}+91=0 \\
& W \geq 0 \\
& W_{3,3}=1
\end{aligned}
$$

Note that the problem in expression (20) leads to a linear objective. This is subjected to use of equalities and inequality of a linear matrix. This type of SDP problem can be solved using CVX tool in polynomial time [16].

4) Complexity analysis: The majority common techniques for resolving SDP issues of modest sizes are IPMs e.g. DSDP [51], SeDuMi [52], SDPA [53], etc. whose computational complexities are polynomial. Semi-definite programs of realistic size may be resolved in polynomial time within any precise precision by IPMs which are iterative algorithms using Newton-like techniques to produce search directions for finding an estimated resolution to the nonlinear system. As the IPMs converge vary fast and precised best solution is attained within a polynomial number of iterations. 
(IJACSA) International Journal of Advanced Computer Science and Applications,

TABLE I. PERFormance AND COMPLEXITY COMPARISON OF MIMO DETECTORS

\begin{tabular}{|c|c|c|c|c|c|c|c|}
\hline Tech & Scheme & Mult. Ant & CSI & BW & Rx Compl. & Benefits & Remarks \\
\hline \multirow{3}{*}{ SM } & V-BLAST [29] & $\mathrm{Tx} \& \mathrm{Rx}(\mathrm{N} / \mathrm{M})$ & $\mathrm{Rx}$ & $\mathrm{N}$ & Moderate & MUX & \multirow{3}{*}{$\begin{array}{c}\text { Moderate complexity, Improved performance } \\
\text { and diversity order }\end{array}$} \\
\hline & D-BLAST & - & - & $\mathrm{N}$ & Moderate & MUX \& DIV & \\
\hline & Turbo-BLAST & Tx \& Rx & - & N/W & Moderate & MUX \& DIV & \\
\hline \multirow{11}{*}{ SD } & MRC & $\mathrm{Rx}$ & $\mathrm{Rx}$ & $\mathrm{N}$ & Low & DIV & \multirow{3}{*}{$\begin{array}{l}\text { Low complexity and Improved performance } \\
\text { and diversity order }\end{array}$} \\
\hline & OSTBCs & Tx (Rx optional) & - & $\mathrm{N}$ & Low & DIV & \\
\hline & STBCs & - & - & $\mathrm{N}$ & Low & DIV & \\
\hline & Linear dispersion codes & - & - & $\mathrm{N}$ & Moderate & DIV and/or MUX & Moderate complexity, \\
\hline & ST-IDM & - & - & N/W & Moderate & DIV & Improved performance. \\
\hline & TR-STBC & - & - & $\mathrm{W}$ & Moderate/high & DIV & \multirow{4}{*}{$\begin{array}{l}\text { High complexity, Improved performance } \\
\text { and diversity order. }\end{array}$} \\
\hline & STTCs & - & - & $\mathrm{N}$ & Moderate/high & DIV \& COD & \\
\hline & Delay diversity & - & - & $\mathrm{N}$ & Moderate/high & DIV & \\
\hline & STTCs & - & - & $\mathrm{N}$ & high & DIV \& COD & \\
\hline & Differential ST schemes & - & - & $\mathrm{N}$ & Varies & DIV (\& COD) & Quasi-ML performance and diversity order, \\
\hline & SF/ STF codes & - & No CSI & $\mathrm{W}$ & Varies & DIV (\& COD) & Low average complexity, High complexity(worst-case), \\
\hline \multirow{3}{*}{ SA } & Rx beamforming & $\mathrm{Rx}$ & $\mathrm{Rx}$ & N/W & Low & ANT & \multirow{2}{*}{$\begin{array}{c}\text { Low complexity and Improved performance } \\
\text { and diversity order. }\end{array}$} \\
\hline & Tx beamforming & Tx & Tx \& & N/W & Low & ANT & \\
\hline & Limited feedback schemes [54] & Tx $(\& \mathrm{Rx})$ & RxTx (lim.) \& Rx & $\mathrm{N}$ & Varies & ANT \& DIV/MUX & High complexity(worst-case) \\
\hline
\end{tabular}

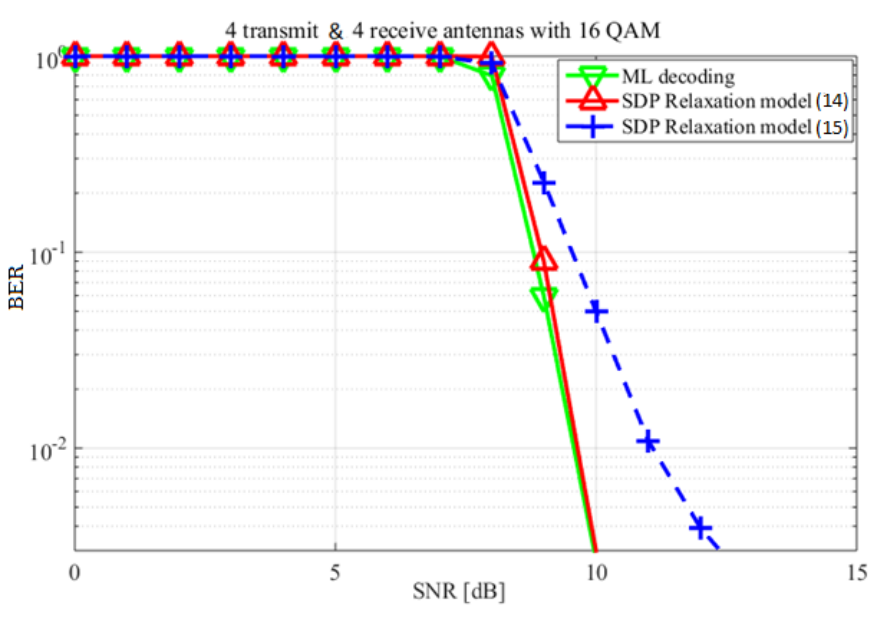

Fig. 10. Complexity comparison.

In our numerical analysis, addition of the non-negativity limitation raises the computational complexity of the using DSDP and SDPA for solving (14), and SeDuMi is implemented for solving (15). Analysis for the worst case complexity of solving models (15) and (16) by IPMs is presented here. The SDP model is devised as a typical linear cone program using slack variables addition for solving relaxation (16) and the linear conic issue using the optimization software SeDuMi [50]. The extra inequality constraints construct the model in (15) significantly sturdy than the model in (15), however too further hard to resolve. The issue in (16) is tractable as the problem sizes of our concern are modest considering a tradeoff among the strength of the bounds and the computational. The randomization process performed here to reinforce the bound achieved is insignificant. Though, utilizing the structure and sparsity feature of semi-definite programs may be vital to the proficient computation of their solution. Every constraint matrices in relaxation models (15) and (16) are rank-one reducing the complexity of interior point algorithms for positive semidefinite programming converging linearly resulting reduction in computation time and memory needs. Fig. 10 depict the performance of (15) and (16).

Various MIMO detectors have different performance-andcomplexity profiles having pros and cons. It seems to be a good time now, after reviewing the state of the art, to establish some comparison amongst all these methods as shown in Table I. We studied a qualitative comparison of the performance and complexity features of the MIMO detectors, and then reviewed their analytical performance and complexity results We then, extended that table (Table I), which depicts the whole picture, listing the proposals strength points and eventual drawbacks. As we can see from Table I, not all methods have the same level of technological consolidation, particularly in terms of signaling and essential necessities.

\section{Simulation EnVironment And Performance ANALYSIS}

The software tool CVX is used for the performance analysis of the optimal MIMO detection approaches based on SDP. It is modeling tool built on top of MATLAB. It is powerful tool for modeling a prototype and algorithms incorporating convex problems using DCP method [45].

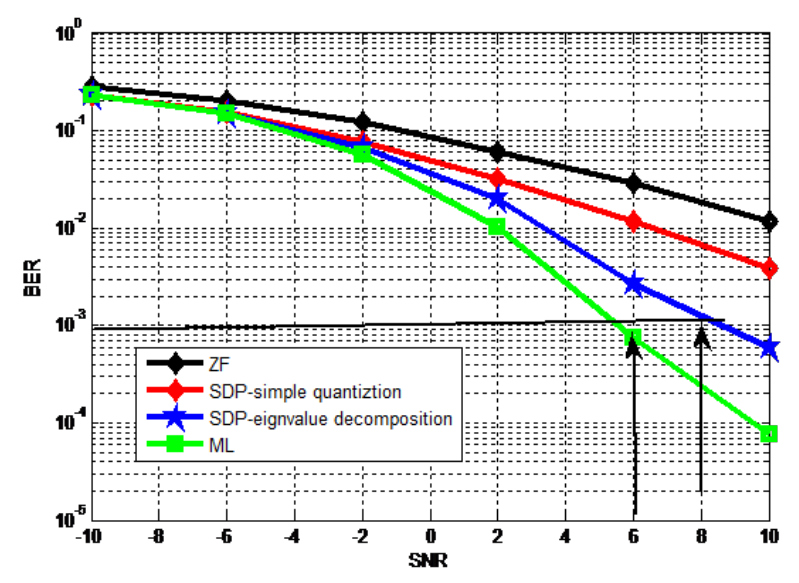

Fig. 11. BER Performance of selected MIMO hard detectors in a $3 \times 3$ systems using 16-QAM signal constellation in a flat-fading channel.

Extensive simulations were run to evaluate and analyze the performance of the quasi optimal MIMO detection approach based on SDP. The results in Fig. 11 depict BER versus SNR for the $3 \times 3$ MIMO system using 16-QAM $\left(16^{3} \approx 4096\right)$ in a flat fading channel. For comparison, we have simulated the optimal and linear detectors. However, all the detectors have same performance from $-10 \mathrm{~dB}$ to $-4 \mathrm{~dB}$ due to high noise effect; 
it is observed that as SNR increases, noise effect decreases and BER performance improves linearly. The optimal detector ML achieves a BER of $10^{-2}$ at an SNR of $2 \mathrm{~dB}$ and BER of $10^{-3}$ at an SNR of $6 \mathrm{~dB}$, by examining all possible transmitted vectors. Suboptimal approach i.e. SDP-simple quantization in a complex system achieves BER of $10^{-2}$ at an SNR of $6 \mathrm{~dB}$ and SDP-Eigen-value decomposition in a complex system achieves BER of $10^{-3}$ at an SNR of $8 \mathrm{~dB}$. ZF achieves BER of $10^{-2}$ at a SNR $10 \mathrm{~dB}$. Here, its BER degraded due to noise amplification. It is easy to see the advantage of the SDR detectors over the ML and ZF detectors. However, SDPEigen-value decomposition approximation technique achieved considerable reduction in complexity at the cost of only $2 \mathrm{~dB}$.

The performance of optimal detector in term of probability is good as compared to simple detector. In [15], the author discussed the performance of different detectors exist in CDMA and SDR detector for higher order QAM constellation in [9] in the context of the MIMO channel. For complexity, simple and closed-form expressions are infrequent. The more convenient way is to model it with increasing complexity with $\mathrm{m}$. The detectors complexity specified through cubic $C(m)$ and is supposed to be in $O(f(m))$, for few function $f(m)$, and $C(m) \leq c f(m)$ for $m \geq M$ where $c$ and $M$ are constants [44]. The complexity of a detector in $O(p(m))$ for few polynomial $p(m)$ the detector is assumed to be polynomial complex one. The complexity $C(m)$ rates are bounded by $O($.$) . Still, polynomial complexity detectors$ are normally considered to be efficient, and the complexity of most polynomial detector is relatively small in practice [44]. Additionally, the complexity measures are obtained through simulations to analyze system performance or design.

The complexity of generic SDP approach for non-convex problem (11a)-(11f) is cubic $O(N 6.5)$ [9]. If relax rank-1 constraints as in (11f) just relax such as (12a)-(12e) and solve diagonal elements in $\mathrm{W}$, then non convex problem is converted into convex problem. Hence the complexity of SDP approach for convexified problem is roughly cubic $O(N 3.5)$, where $N=2 M+1$, while $\mathrm{M}$ is the number of QAM symbols[9].

The simulation results in Fig. 12 depicts BER versus SNR of SDP-simple quantization approximation technique in different system configurations using 16-QAM constellation in a flat fading channel. The performance of SDP-simple quantization in computationally complex system improves with increasing in number of transmit and received vectors. However, suboptimal approach $(4 \times 4)$ SDP achieved a BER of $10^{-2}$ at an SNR of $5 \mathrm{~dB},(3 \times 3)$ SDP achieved a BER of $10^{-2}$ at an SNR of $6 \mathrm{~dB}$ and $(2 \times 2)$ SDP achieved a BER of $10^{-2}$ at an SNR of $9 \mathrm{~dB}$.

The results in Fig. 13 depict BER versus SNR for the different antenna configuration using 16-QAM $\left(16^{3} \approx 4096\right)$ in a flat fading channel. However, SDP-simple quantization approximation technique in $(2 \times 4)$ MIMO system achieved BER of $10^{-3}$ at an SNR of $2 \mathrm{~dB}$; in $(3 \times 4)$ MIMO system achieved a BER of $10^{-3}$ at an SNR of $6 \mathrm{~dB}$; and in $(4 \times 4)$ MIMO system achieves a BER of $10^{-2}$ at an SNR of $2 \mathrm{~dB}$. However, SDP-simple quantization in $(2 \times 4)$ and $(3 \times 4)$ MIMO systems performance is improved.

The results in Fig. 14 depict BER versus SNR for the $3 \times 3$ system using 16-QAM $\left(16^{3} \approx 4096\right)$ in flat fading channel. For

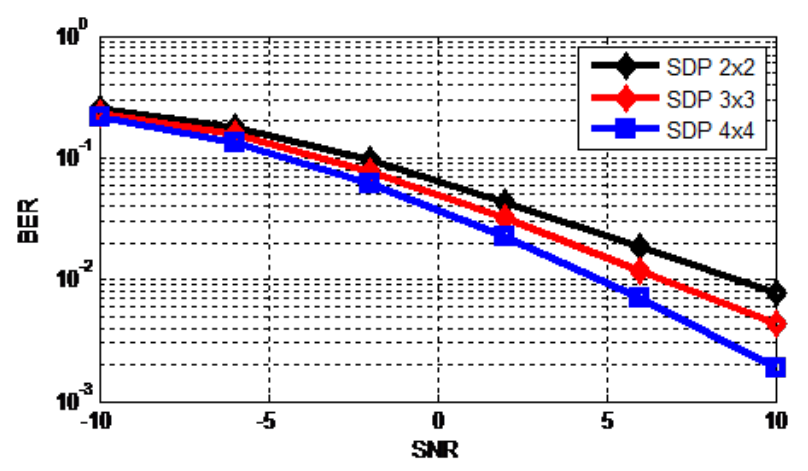

Fig. 12. BER Performance of different system configurations MIMO systems using16- signal constellation in a flat fading channel.

comparison, we have simulated the suboptimal detector with full complexity and near optimal detector with different search spaces. However, suboptimal and near optimal detectors have same performance from $-10 \mathrm{~dB}$ to $-4 \mathrm{~dB}$ due to high noise effect. It is observed that as SNR increases noise effect decreases and BER performance improves linearly.

However, sub-optimal detector SDP-simple quantization approximation technique achieved a BER of $10^{-2}$ at an SNR of $6 \mathrm{~dB}$ in computationally complex system. ZFML with 16 vector search space at 2.5 neighbor size with $|2|^{M t}$ reduction rate achieves a BER of $10^{-2}$ at an SNR of $13 \mathrm{~dB}$; ZFML with 64 vector search space at 4 neighbor size with $|4|^{M t}$ reduction rate achieved a BER of $10^{-2}$ at an SNR of 10dB; ZFML with 125 vector search space at 5 neighbor size with $|5|^{M t}$ reduction rate achieves a BER of $10^{-2}$ at an SNR of 9dB; and ZFML with 343 vector search space at 7 neighbor size with $|7|^{M t}$ reduction rate achieved a BER of $10^{-2}$ at an SNR of $7 \mathrm{~dB}$. ZFML computational complexity is bounded by maintaining small search areas, while performance is maximized by relaxing this constraint and increasing the cardinality of the search space.

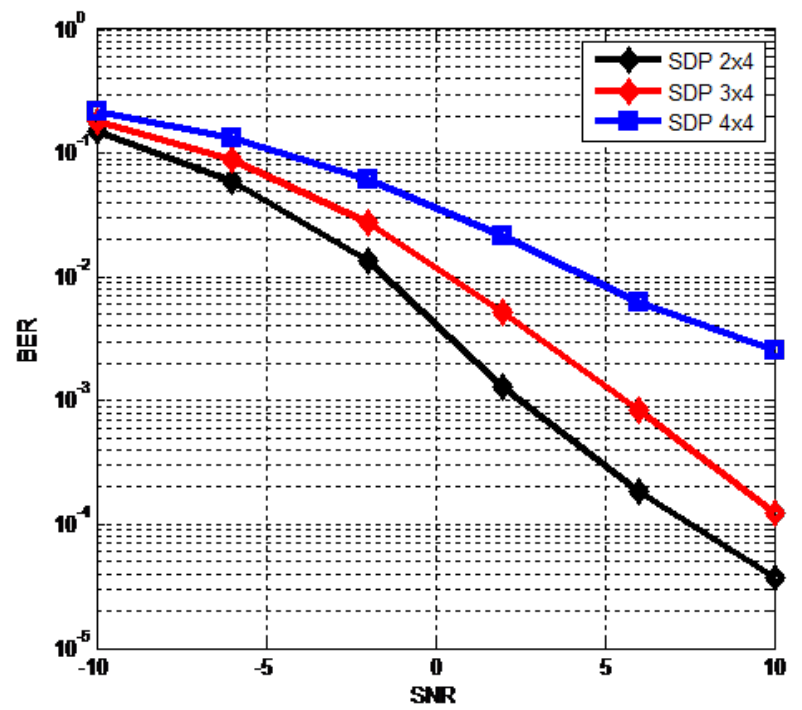

Fig. 13. BER Performance of different Antenna Configurations MIMO Systems using 16-QAM signal constellation in a flat fading channel. 


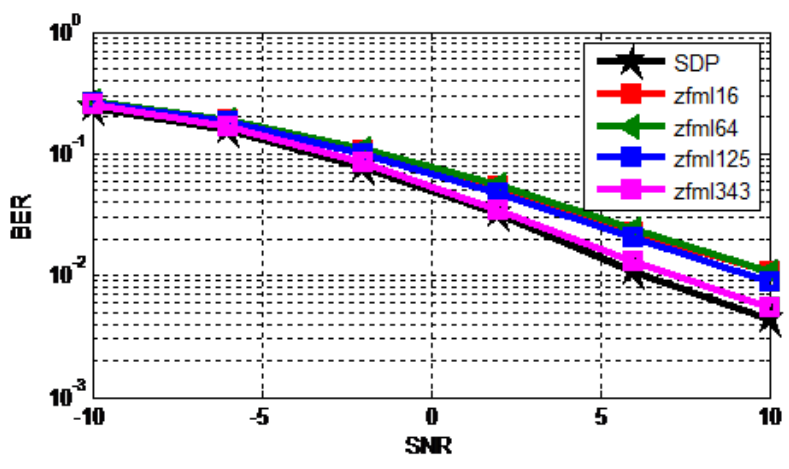

Fig. 14. BER Performance of SDP and ZFML MIMO hard Detectors in a $3 \times 3$ Systems using 16- QAM signal constellation in a flat fading channel.

However, SDP-simple quantization approximation technique achieved considerable performance in a computationally complex problem $\left(16^{3} \approx 4096\right.$ vectors $)$ as compared to the ZFML detector in a less computationally complex problem $|7|^{M t}$ ( $7^{3} \approx 343$ vectors). Therefore, we find the computationally efficient SDR detector as a competitive detector in comparison to other near-optimal methods.

Compared to SDP that performs a coarse search over the complete search space the ZFML used a reduced constellation, therefore its computational complexity is $\left(M / M_{n}\right)^{M}$. Where $M$ is the constellation size, $M_{n}$ is the neighbors list and $M_{t}$ is the number of transmitters.

\section{Conclusion And Future Discussion}

The presented work aimed to analyze the efficiency of MIMO detection approaches both in terms of BER performance and computational complexity. Specifically, the work is focused on performance evaluation and comparison of two heuristic suboptimal detection algorithms previously proposed in literature, namely, the ZFML and the Semi-definite relaxation detectors. The presented simulation results are relating to the performance of the two algorithms including the comparison with linear and optimal detection schemes for MIMO systems. Most important result is that while it was proven by the analytical results that the ZFML detector is better in large search space, which increases the computational complexity, the SDR detector is computationally efficient detector in same scenario. Possible future work is to analyze SDP and ZFML, BER/computational complexity performance in MIMO system using higher order constellation in a flat fading/Rayleigh channel.

\section{REFERENCES}

[1] Kapila C. Wavegedara, Gaurav Bansal, Wireless Communications: Trends and Challenges chapter from book Next-Generation Wireless Technologies: 4G and Beyond (pp.3-5) Jan 2013.

[2] Cisco Visual Networking Index: Global MobileData Traffic Forecast Update, 2015-2020, WhitePaper, Cisco, Feb. 2016. [Online]. Available:http://www.cisco.com/c/en/us/solutions/collateral/serviceprovider/visual-networking-index-vni/white paper c11-520862.pdf

[3] D. Mcqueen, The momentum behind LTE adoption, IEEE Communications Magazine, vol. 47, no. 2, pp. 4445, Feb. 2009.

[4] M. El-Sayed, A. Mukhopadhyay, C. Urrutia-Valdes, and Z. J.Zhao, Mobile data explosion: monetizing the opportunity through dynamic policies and QoS pipes, Bell Labs Technical Journal,vol. 16, no. 2, pp. 7999 , Sep. 2011. [Online]. Available: http://dx.doi.org/10.1002/bltj.20504
[5] B. Han, P. Hui, V. S. A. Kumar, M. V. Marathe,J. Shao, and A. Srinivasan,Mobile data offloading through opportunistic communications and social participation, IEEETransacti ons on Mobile Computing, vol. 11, no. 5, pp. 821834,May 2012.

[6] G. Lawton, Machine-to-machine technology gears up for growth,IEEE Computer Magazine, vol. 37, no. 9, pp. 1215, Sep. 2004.

[7] G. Wu, S. Talwar, K. Johnsson, N. Himayat, and K. D. Johnson, M2M:from mobile to embedded internet,IEEE Communications Magazine,vol. 49, no. 4, pp. 3643, Apr.2011.

[8] Y. Zhang, R. Yu, S. Xie, W. Yao, Y. Xiao, and M. Guizani, HomeM2M networks: architectures, standards, and QoS improvement, IEEECommunications Magazine, vol. 49, no. 4, pp. 4452, Apr. 2011.

[9] Kuldeep Kumar, A comparison of different detection algorithms in a MIMO system, journal of advanced engineering sciences and technologies, volume no. 7, issue No 2, 301 304, 2011.

[10] Jun Zhang, Runhua Chen Networked MIMO with clustered linear precoding Wireless Communications, IEEE Transactions, volume: 8, Issue: 4, page(s): 1910 1921, August 2008.

[11] Sajid Bashir, M. Naeem, An Application of Univariate Marginal Distribution Algorithm in MIMO Communication Systems. International journal of communication system, volume 23, issue 1, pages 109-124, January 2010.

[12] Adnan Ahmed Khan, Symbol Detection techniques in Spatial Multiplexing systems, PhD Thesis December 2008.

[13] Xiaodong Li, Howard C, Reduced-Complexity Detection Algorithms for Systems Using Multi-Element Arrays, IEEE telecommunication, volume: 2 page(s): 1072 1076, 2000.

[14] Wing-Kin Ma, Chao-Cheng Su, The Equivalence of Semidefinite Relaxation MIMO Detectors for Higher-Order QAM, IEEE journal of selected topics in signal processing, vol. 3, no. 6, December 2009.

[15] P.H. Tan and L.K. Rasmussen, The application of semi-definite programming for detection in CDMA, IEEE Journal on Selected Areas in Communications, 19(8):1442 1449, August 2001.

[16] Ami Wiesel, Eldar Y, Shamai S, Semidefinite relaxation for detection of 16-QAM signaling in MIMO channels, IEEE Signal Processing Letters, 12(9):653656, 2005.

[17] Nicholas D Sidiropoulos, A Semidefinite Relaxation Approach to MIMO Detector for High-order QAM constellations, IEEE Signal Processing letters, vol.13, page NO.9, September 2006.

[18] Andrea Goldsmith, MIMO Wireless Communications, Cambridge University Pr,08-Aug-2005.

[19] C. E. Shannon, A mathematical theory of communication, The BellSystem Technical Journal, vol. 27, no. 3, pp. 379423, Jul. 1948.

[20] D. Gesbert, S. V. Hanly, H. Huang, S. Shamai (Shitz), O. Simeone,and W. Yu, Multi-cell MIMO cooperative networks: a new look at interference, IEEE Journal on Selected Areas in Communications, vol. 28, no. 9, pp. 13801408, Dec. 2010.

[21] S. Shamai (Shitz) and A. D. Wyner, Information-theoretic considerationsfor symmetric, cellular, multiple-access fading channels Part I,IEEE Transactions on Information Theory, vol. 43, no. 6, pp. 18771894, Nov. 1997.

[22] Attainable throughput of an interference-limited multiple-inputmultipleoutput (MIMO) cellular system,, IEEE Transactions on Communications,vol. 49, no. 8, pp. 13071311, Aug. 2001.

[23] S. Verdu, Computational complexity of optimum multiuser detection,Algorithmica, vol. 4, no. 1-4, pp. 303312, Jun. 1989. [Online].Available: http://dx.doi.org/10.1007/BF01553893

[24] D. Micciancio, The hardness of the closest vector problem withpreprocessing, IEEE Transactions on Information Theory, vol. 47,no. 3, pp. 12121215, Mar. 2001.

[25] L. C. Hui and K. B. Letaief, Successive interference cancellationfor multiuser asynchronous DS/CDMA detectors in multipath fadinglinks, IEEE Transactions on Communications, vol. 46, no. 3, pp. 384391, Mar. 1998

[26] X. Wang and H. V. Poor, Wireless Communication Systems: AdvancedTechniques for Signal Reception, 1st ed. Upper Saddle River, NJ,USA: Prentice Hall, 2009.

[27] R. H. Roy and B. Ottersten, Spatial division multiple access wirelesscommunication systems, U.S. Patent 5515 378, May 7, 1996. 
[28] M. P. Lotter and P. van Rooyen, Space division multiple access forcellular CDMA, in Proc. IEEE 5th International Symposium on SpreadSpectrum Techniques and Applications (ISSSTA98), Sun City, South Africa, Sep. 1998, pp. 959964.

[29] G. D. Golden, G. J. Foschini, R. A. Valenzuela, and P. W. Wolniansky,Detection algorithm and initial laboratory results using VBLASTspace-time communication architecture, Electronics Letters, vol. 35,no. 1, pp. 1416, Jan. 1999.

[30] H. Jafarkhani, A quasi-orthogonal space-time block code, IEEETransactions on Communications, vol. 49, no. 1, pp. 14, Jan. 2001.

[31] H. Jafarkhani, Space-Time Coding: Theory and Practice. Cambridge University Press, 2005.

[32] Z.-Q. Luo, W.-K. Ma, A. M.-C. So, Y. Ye, and S. Zhang, Semidefiniterelaxation of quadratic optimization problems, IEEE Signal ProcessingMagazine, vol. 27, no. 3, pp. 2034, May 2010.

[33] Z.-Q. Luo and W. Yu, An introduction to convex optimization forcommunications and signal processing, IEEE Journal on SelectedAreas in Communications, vol. 24, no. 8, pp. 14261438, Aug. 2006.

[34] L. Vandenberghe and S. Boyd, Semidefinite programming, SIAMReview, vol. 38, no. 1, pp. 4995, Mar. 1996.

[35] S. Boyd and L. Vandenberghe, Convex Optimization. New York, USA:Cambridge University Press, 2004

[36] C. Helmberg, F. Rendl, R. J. Vanderbei, and H. Wolkowicz, Aninteriorpoint method for semidefinite programming, SIAM Journalon Optimization, vol. 6, pp. 342361, 1996.

[37] Z.-Q. Luo and W. Yu, An introduction to convex optimization forcommunications and signal processing, IEEE Journal on SelectedAreas in Communications, vol. 24, no. 8, pp. 14261438, Aug. 2006.

[38] P. H. Tan and L. K. Rasmussen, The application of semidefiniteprogramming for detection in CDMA, IEEE Journal on Selected Areasin Communications, vol. 19, no. 8, pp. 14421449, Aug. 2001.

[39] W.-K. Ma, C.-C. Su, J. Jalden, T.-H. Chang, and C.-Y. Chi, Theequivalence of semidefinite relaxation MIMO detectors for higher-orderQAM, IEEE Journal of Selected Topics in Signal Processing, vol. 3,no. 6, pp. 10381052, Dec. 2009.

[40] X. Wang, W.-S. Lu, and A. Antoniou, A near-optimal multiuserdetector for CDMA channels using semidefinite programming relaxation, in Proc. IEEE International Symposium on Circuits and Systems(ISCAS01), Sydney, NSW, Australia, May 2001, pp. 298301.

[41] A near-optimal multiuser detector for DS-CDMA systems usingsemidefinite programming relaxation, IEEE Transactions on SignalProcessing, vol. 51, no. 9, pp. 24462450, Sep. 2003.
[42] W.K. Ma, T.N. Davidson, Quasi-maximum-likelihood multiuser detection using semi-definite relaxation with application to synchronous CDMA, IEEE Transaction Signal Processing, 50(4):912922, April 2002.

[43] P.H. Tan and L.K. Rasmussen, The application of semi-definite programming for detection in CDMA, IEEE Journal on Selected Areas in Communications, 19(8):14421449, August 2001.

[44] Shaoshi Yang, Lajos H, Semi-definite Programming Relaxation BacedVirtulally Antipodal Detection for Gray Coded 16-QAM MIMO Signaling, IEEE Communication, 978-1-4244-9268-8, November 2011.

[45] M. Grant, S. Boyd, Disciplined convex programming, Global Optimization, Springer, pages 1552102006.

[46] L. Vandenberghe, S. Boyd, Semi-definite programming, SIAM Review, 38:4995, 1996.

[47] C. Helmberg, F. Rendl, An interior-point method for semi-definite programming. SIAM Journal on Optimization, 6:342-361, 2005.

[48] Y.E. Nesterov, Quality of semi-definite relaxation for nonconvex quadratic optimization, Technical Report, University Catholique, Belgium, 1997.

[49] Mobasher, M. Taherzadeh, R. Sotirov, and A. K. Khandani, An Efficient Quasi-Maximum Likelihood Decoding for Finite Constellations, Department of E\&CE, University of Waterloo, Tech. Rep. UW-E\&CE 2005-01, 2005, available via the WWW site at http : //www.cst.uwaterloo.ca/a min

[50] J. Sturm, .Using sedumi 1.02, a matlab toolbox for optimization over symmetric cones,. Optimization Methods and Software, vol. 11-12, pp. 625.653, 1999.

[51] S. J. Benson and Y. Ye, .DSDP5 User Guide The Dual-Scaling Algorithm for Semide-nite Programming,. Mathematics and Computer Science Division, Argonne National Laboratory, Argonne, IL, Tech. Rep. ANL/MCS-TM-255, 2004, available via the WWW site at http : //www.mcs.anl.gov/benson.

[52] J. Sturm, .Using sedumi 1.02, a matlab toolbox for optimization over symmetric cones,. Optimization Methods and Software, vol. 11-12, pp.625.653, 1999.

[53] M. Kojima, K. Fujisawa, K. Nakata, and M. Yamashita, .SDPA(SemiDenite Programming Algorithm) Users Mannual Version 6.00,. Dept. of Mathematical and Computing Sciences, Tokyo Institute of Technology, 2-12-1 Oh-Okayama, Meguro-ku, Tokyo, 152-0033, Japan, Tech. Rep., 2002, available via the WWW site at http://sdpa.is.titech.ac.jp/SDPA/.

[54] J. C. Roh and B. D. Rao, Design and analysis of MIMO spatial multiplexing systems with quantized feedback, IEEE Trans. Signal Processing, vol. 54, no. 8, pp. 28742886, Aug. 2006. 